\title{
SPONTANEOUS PNEUMOTHORAX COMPLICATING ARTIFICIAL PNEUMOPERITONEUM
}

\author{
BY
}

\author{
E. G. SITA LUMSDEN
}

From the London Hospital Annexe, Brentwood, Essex

The spontaneous development of a pneumothorax during the course of treatment with an artificial pneumoperitoneum has only rarely been reported. In most instances the pneumothorax has been ascribed to air entering the mediastinum from the abdominal cavity by one of the diaphragmatic hiatuses, and thence rupturing into the pleural sac, in nearly every case on the right side. A pneumothorax may be induced accidentally on either side when the lower intercostal route is employed for the induction or maintenance of a pneumoperitoneum, and such accidents are not uncommon (Banyai, 1946); for this reason the intercostal route has largely been abandoned.

Banyai, a pioneer of pneumoperitoneum treatment, in his experience dating from 1931 has recorded only one case of spontaneous as distinct from accidental pneumothorax (Banyai and Jurgens, 1940 ; Banyai, 1946). A man of 53, with a pneumoperitoneum which had been maintained for a year, was given his usual refill of $1,000 \mathrm{ml}$. through a point well below and to the left of the umbilicus, the final pressures being $+12+10$. Shortly afterwards he suddenly developed a rightsided pneumothorax and his pneumoperitoneum rapidly deflated. Mitchell and others (1947) do not mention this complication in their review of 710 cases of artificial pneumoperitoneum, nor does any reference to it occur in the writings of Stokes (1938), Clifford-Jones and MacDonald (1943), Rilance and Warring (1944), Edwards and Logan (1945), Keers (1947, 1948), or Trimble (1948), all of whom have published large series of cases.

Smith (1943) described a fatal case of bilateral spontaneous pneumothorax following the induction of a pneumoperitoneum for diagnostic purposes. Death occurred shortly after induction, and at necropsy the lungs were found collapsed and there were several small holes in both halves of the diaphragm connecting peritoneal and pleural cavities. Simmonds (1946), discussing air embolism and mediastinal emphysema occurring during the course of pneumoperitoneum therapy, tells of a woman aged 29 who developed spontaneously a right pneumothorax after her pneumoperitoneum had been maintained for eight months. He attributed the accident to air entering the mediastinum from the abdomen and thence bursting into the pleural cavity.

Laird (1945) observed at thoracoscopy subpleural air bubbles on the diaphragm in four patients who had both a pneumothorax and a pneumoperitoneum with a paralysed hemidiaphragm. He suggested the possibility of defects in the peritoneal covering of the under-surface of the diaphragm, and this "would explain the occasional case where the air suddenly passes from the abdomen and an unsuspected pneumothorax is found."

Mellies (1939) reported a case of pneumoperitoneum complicated by rupture of an erosive tuberculous lesion of the diaphragm and the establishment of a right-sided pneumothorax.

Vajda (1933) referred to the possibility of air entering the abdominal cavity from the pleural sac owing to the presence of a direct communication between the two, due to a rare congenital defect. Banyai (1933) described two such cases, both in women, in whom indirect pneumoperitoneum followed artificial pneumothorax. $\mathrm{He}$ ascribed the occurrence, however, to the migration of air along one of the mediastinal structures passing through the diaphragm.

\section{Case History}

A woman aged 38 years was admitted to hospital in June, 1948, with symptoms of pulmonary tuberculosis which had been present for nine months. Radiography showed a large cavity in the left upper zone with recent spread of infiltration to the left base and right second interspace. On July 27 she was transferred to the London Hospital Annexe for treatment with streptomycin. Her general condition was only fair ; she was febrile and the sputum contained numerous acid-fast bacilli. The vital capacity was $1,400 \mathrm{ml}$. 

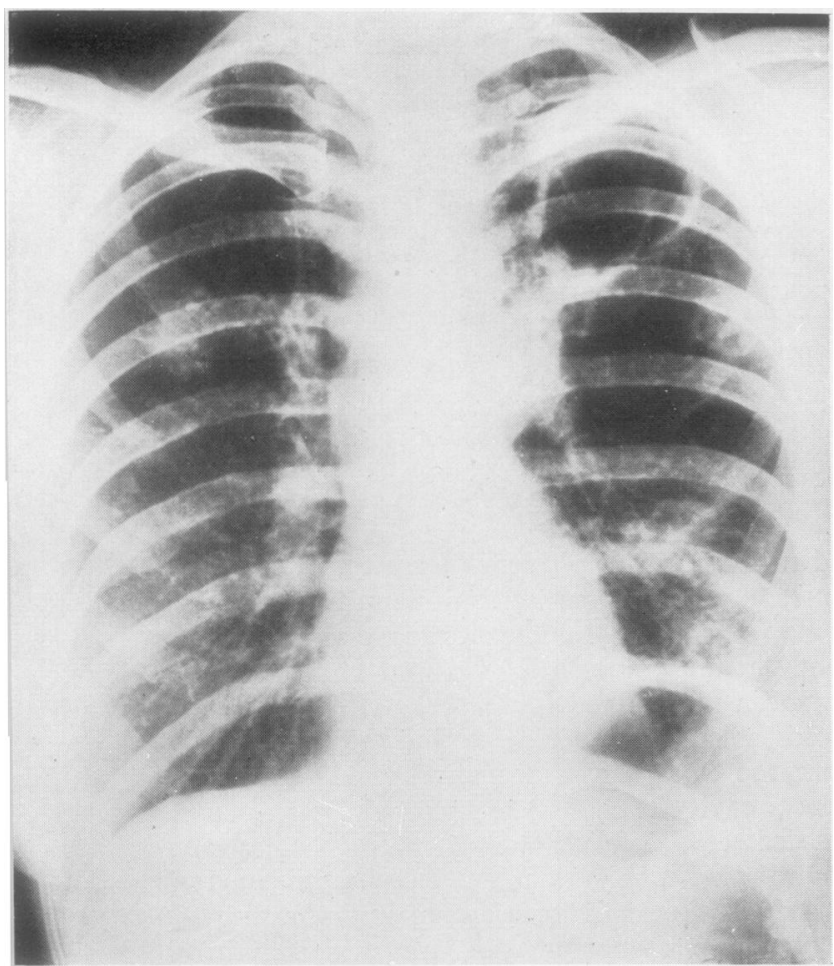

Fig. 1.-Aug. 24, 1948, after left phrenic crush.
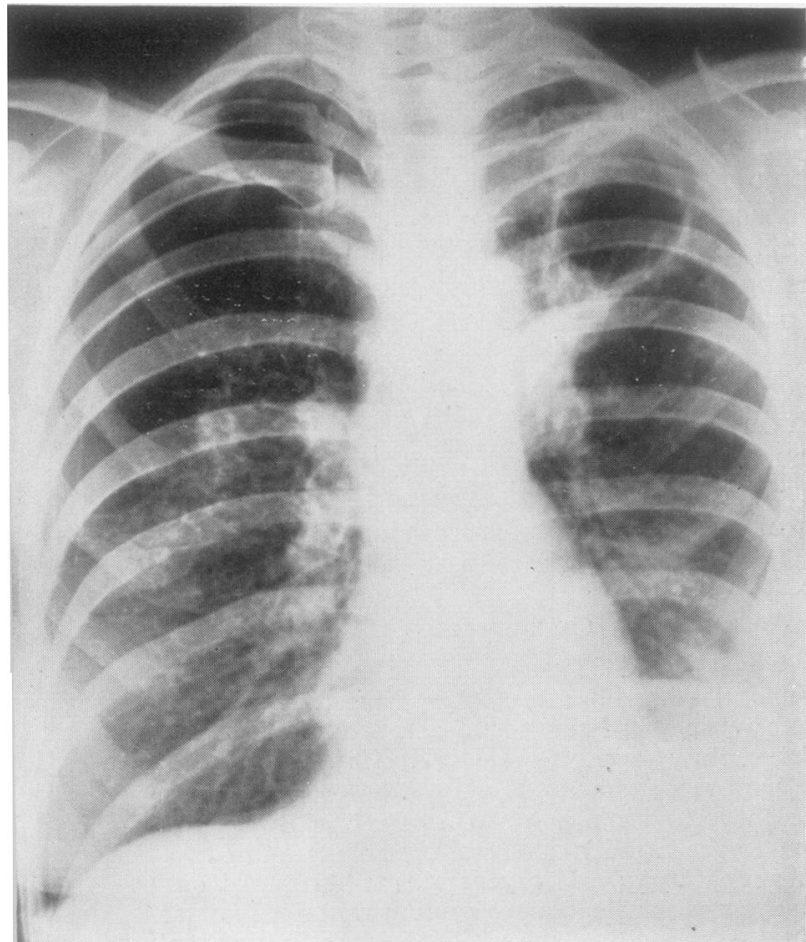

FIG. 3.-Sept. 7, 1948. Pneumothorax persisting after disappearance of air from the peritoneum.

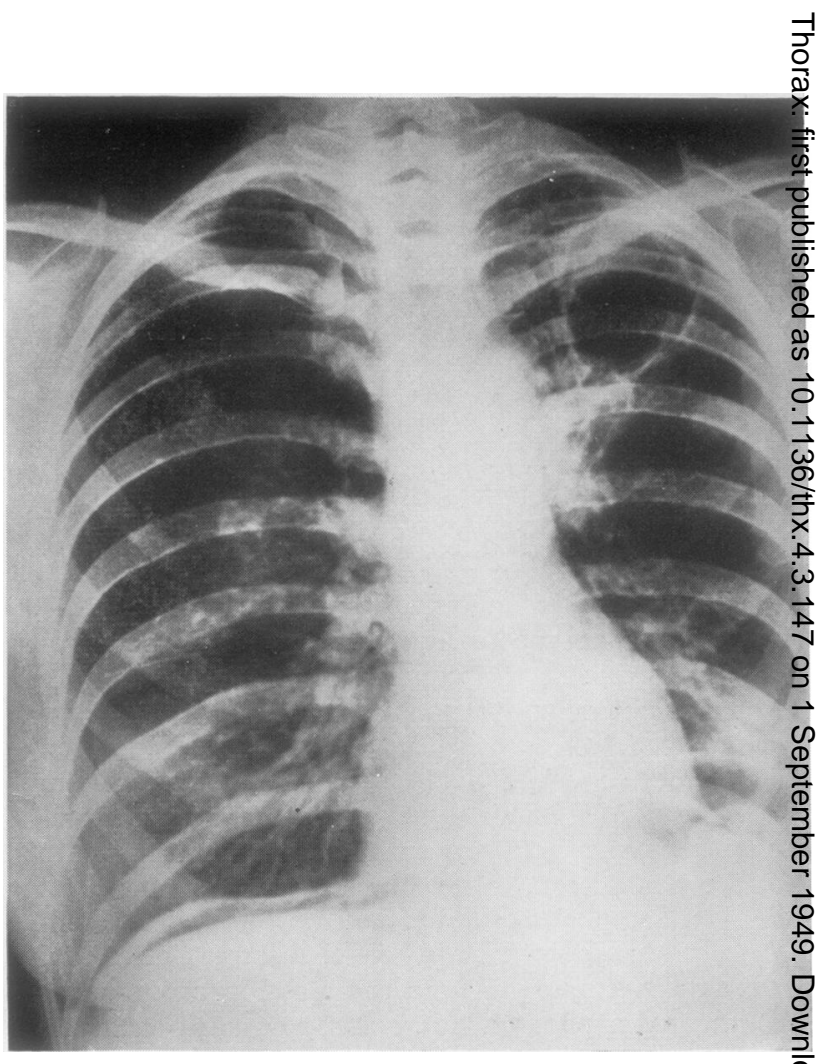

FIG. 2.-Sept. 4, 1948, after induction of pneumoperitoneum Air has entered the right pleura.

Streptomycin was given for two months, and o $\vec{B}$ Aug. 23, 1948, Mr. Vernon Thompson crushed the left phrenic nerve, with a resulting $4 \mathrm{~cm}$. rise, para? lysis, and paradoxical movement of the left cupole (Fig. 1).

Pneumoperitoneum was induced on Aug. 31, 1948 by the technique usually employed at this hospita the meedle being inserted one inch below the lef costal margin at the lateral border of the rectug sheath.

The initial pressure was atmospheric and did no@ fluctuate. Air entered with ease $(800 \mathrm{ml}$.), and the final pressure was +6 ; liver dullness dis? appeared; the epigastric hollow filled and pais was felt in the right shoulder. Clinically there was no evidence of subcutaneous or mediastina e.mphysema.

On Sept. 2 no air could be seen in the abdominà cavity on screening. On Sept. 4 a refill of $1,000 \mathrm{~m} R$ was given, and two hours later a radiograph showed a shallow pneumoperitoneum and an unsuspecte right pneumothorax (Fig. 2). This was probably pres ent when the patient was screened two days pre्ष viously, but was missed. The intrapleural pressures were $-6-1$. Three days later all the air had dis appeared from the abdomen, and the pneumothoras remained unchanged, pressures still being -6 (Fig. 3).

On Sept. 10 the pneumoperitoneum was re-induce $\Phi$ The initial pressures were zero, then $1,000 \mathrm{ml}$. ad was introduced, and the final pressures were +s The initial and final pressures in the pneumothoras at the same time were $-5-1$ and $-4-0$. That evere ing the patient became very breathless, and 700 nat 


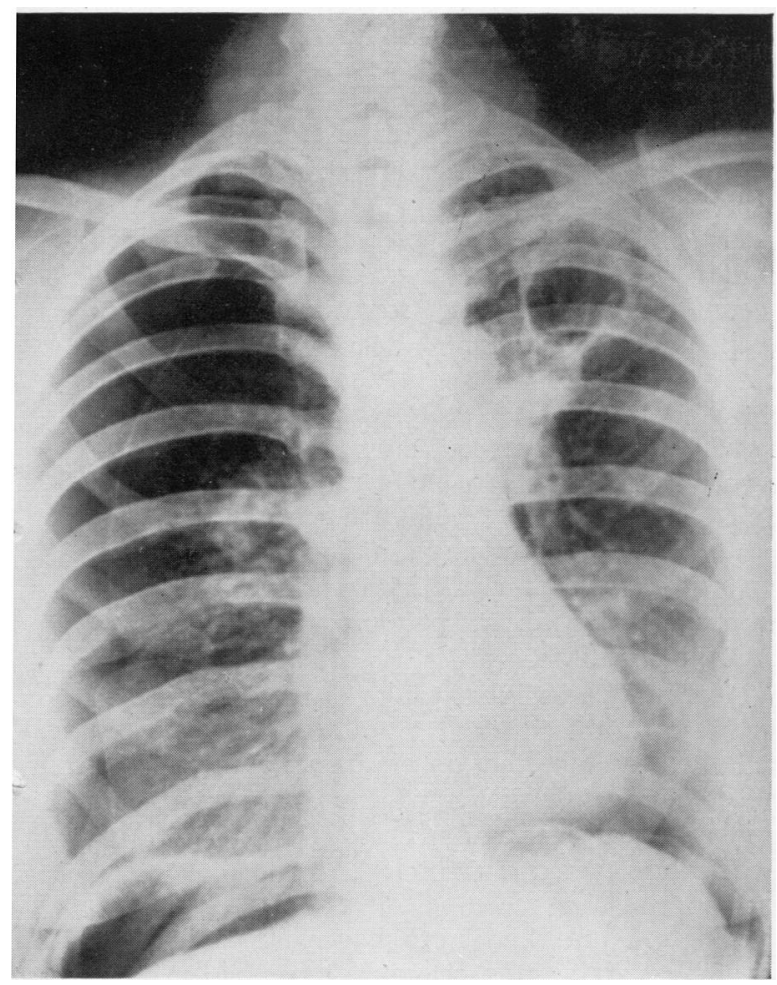

Fig. 4.-Sept. 14, 1948, after refill of pneumoperitoneum. More air has entered the right pleura.

of air had to be aspirated from the chest, pressures before and after being $-6+8$ and $-5-1$.

On Sept. 14, screening having shown that no air remained under the diaphragm, $1,000 \mathrm{ml}$. air were given and a radiograph taken (Fig. 4). Six hours later a further radiograph (Fig. 5) showed very little air remaining in the peritoneal cavity, and screening next day showed that all the air had entered the right ipleural sac.

Radiological examination with an opaque meal showed no evidence of herniation of the stomach through the diaphragm. An attempt was made to ascertain the site of the presumed pleuro-peritoneal communication by posturing. A refill of $1,200 \mathrm{ml}$. iwas given into the peritoneum and the patient turned on her left side. She maintained this posture uninterruptedly, and a week later fluoroscopy showed that a large air bubble still remained trapped under the right hemi-diaphragm lateral to the liver, suggesting that the gap in the diaphragm was situated close to the midline. When she sat up the air rapidly vanished into her chest.

On Oct. $4 \mathrm{Mr}$. V. C. Thompson examined the right pleura through a thoracoscope, after a pneumoperitoneum refill of $1,200 \mathrm{ml}$. had been given. The diaphragm looked normal and there was no obvious defect; the only feature of note was the presence of two subpleural air bubbles about 5 and $3 \mathrm{~mm}$. in diameter. These were watched for 10 minutes but did not grow larger.

An attempt was then made to find out whether a similar pleuro-peritoneal communication existed on the left side, masked possibly by the presence of pleural adhesions. If a pneumothorax could be

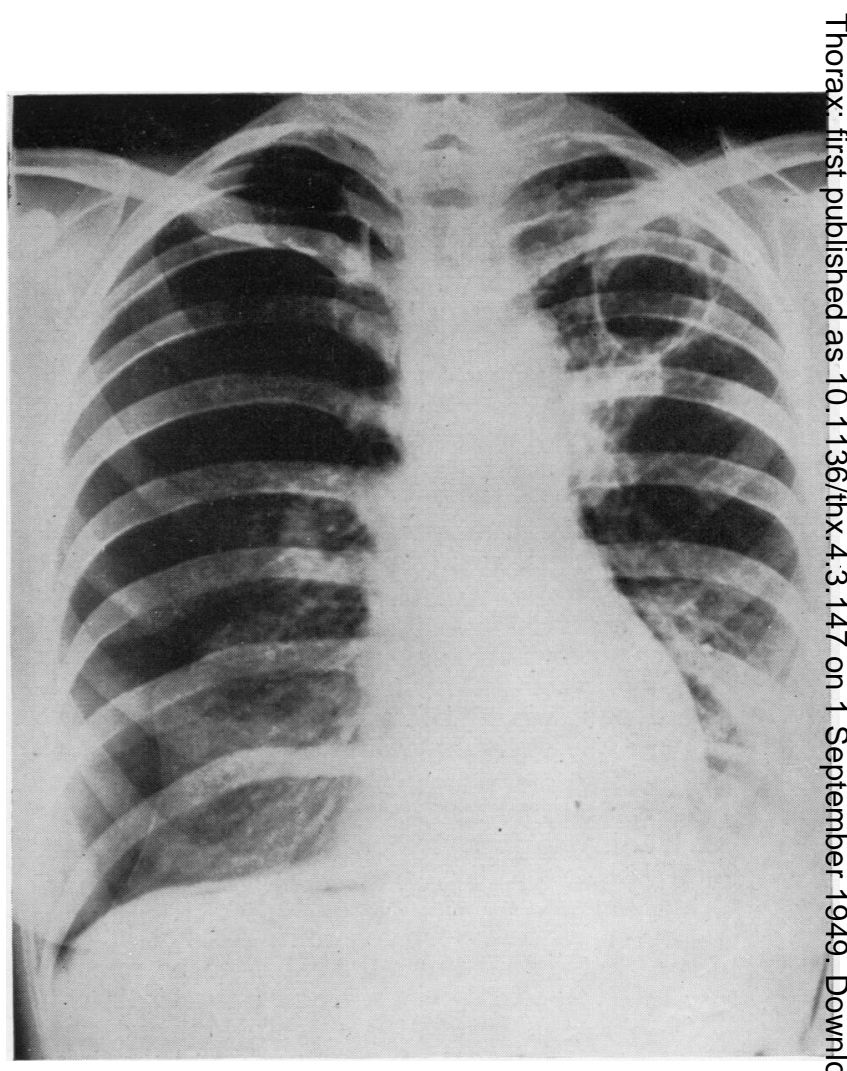

FIG. 5.-Sept. 14, 1948 (six hours after Fig. 4). Very little ad remains in the peritoneum and the right pneumothorax larger.

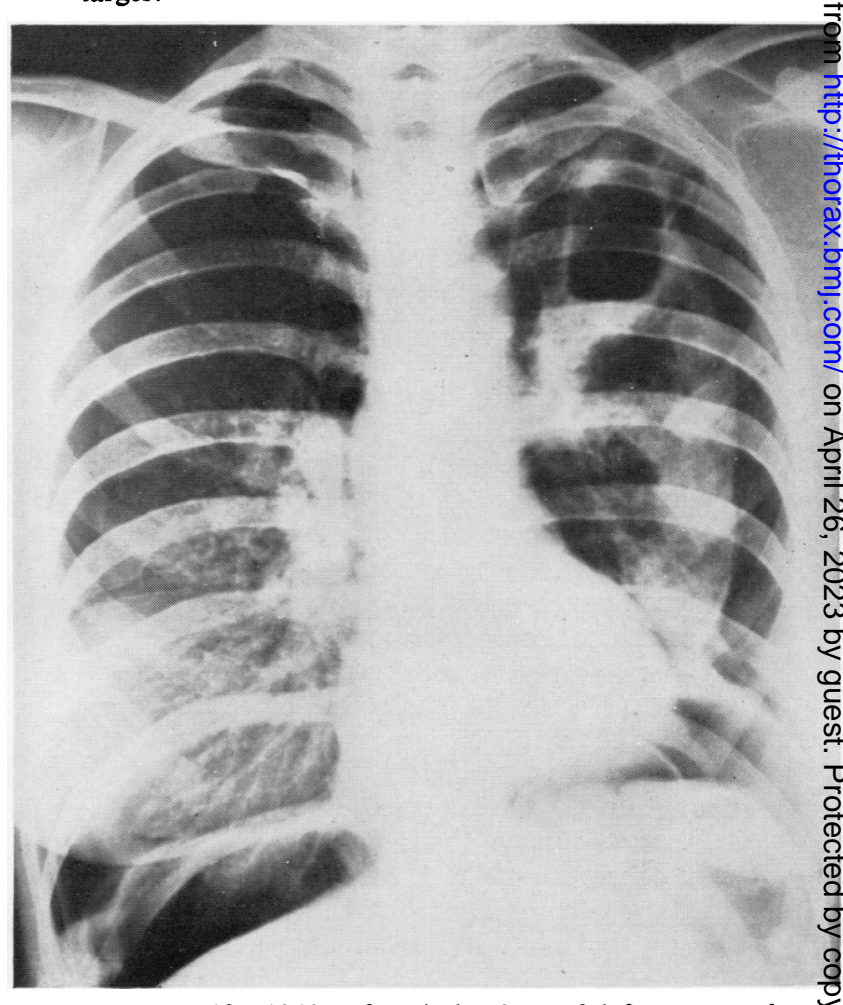

FIG. 6.-Oct. 12, 1948, after induction of- left pneumothora㷴 to demonstrate free left pleural space. 
induced and the lung shown to be free at the base, then clearly no such communication existed on the left side, for there were no subdiaphragmatic adhesions. A left artificial pneumothorax was established, and on screening the left lower lobe was found to be free of the diaphragm; the left pneumothorax did not increase in size after a further pneumoperitoneum refill, whereas the right lung collapsed still further (Fig. 6). No air could be made to pass through the left cupola, whatever position the patient assumed. One thousand $\mathrm{ml}$. air passed through the right hemidiaphragm in less than two hours.

A further attempt was made to demonstrate the site of the communication by instilling $20 \mathrm{ml}$. of radio-opaque oil into the right pleural cavity under the $x$-ray screen. The oil collected in the costophrenic sinus posteriorly, but none could be made to pass into the abdomen whatever position the patient was put into. The lung was accidentally punctured when injecting the oil and a tension pneumothorax developed, with pressures $+1+28$ and gross mediastinal shift to the left. In spite of the high intrapleural pressure no air appears to have passed back into the pneumoperitoneum, which did not increase in size, and several litres of air had to be removed from the pleural cavity. A small lymphocytic effusion followed the instillation of iodized oil, and it was hoped that the pleural reaction would seal the defect in the diaphragm, but when the pneumoperitoneum was re-induced on Dec. 4 with $1,000 \mathrm{ml}$. air the air passed rapidly into the pleural cavity again. The pneumoperitoneum was therefore finally abandoned.

\section{Discussion}

It has unfortunately not been possible to demonstrate incontrovertibly the route taken by the air in passing from the peritoneal to the right pleural cavity. There are several theoretical possibilities.

The first is suggested by Banyai (1946) and by Simmonds (1946) that the air passed through one of the normal hiatuses of the diaphragm into the posterior mediastinum, and then burst through the mediastinal pleura into the pleural cavity on the right side. There are definite objections to such a hypothesis. Air cannot pass through the oesophageal, caval, or aortic hiatuses of the diaphragm from the peritoneal sac unless there is a defect in the parietal peritoneum; such a peritoneal defect would have no embryological basis. In order to reach the mediastinum air would have to be injected extraperitoneally, a not infrequent accident during the course of pneumoperitoneum treatment, but in the present case such an accident does not appear to have occurred at any time. That air can readily reach the pleural cavity from the mediastinum has been shown experimentally by Macklin (1937), and an interesting case of pneumomediastinum following bronchoscopy has been described by Jones (1945). The air was under considerable pressure and a right-sided spontaneous pneumothorax and pneumoperitoneum developed, but how the air reached the pleural and peritoneal sacs was not demonstrated. Two cases of spontaneous pneumothorax following mediastinal emphysema were also reported by Hamman (1939). In Banyai's patient, however, there does not appear to have been any clinical evidence of interstitial emphysema, nor does a skiagram show any air in the mediastinum. In the present instance no symptom or sign, clinical or radiological, of pneumomediastinum was noted at any time.

A second possibility would be the passage of air by diffusion through diaphragm and pleura, facilitated by imperfect development of the muscle and tendinous tissue or paretic thinning of the cupola, as suggested by Laird (1945). In this patient, however, the right leaf of the diaphragm was normal on radiological and thoracoscopic examination and had not been paralysed. The transfer of air always occurred too rapidly to be accounted for by diffusion.

Multiple defects in serosa and muscle as in Smith's (1943) case were excluded by thoracoscopy, as was rupture of an ulcerative tuberculous lesion of the diaphragm.

Finally the possibility remains of the persistence of a patent pleuro-peritoneal cand on the right side. Unfortunately it was not possible to demonstrate directly such a congenital defect, but its presence can be inferred with considerable certainty. A pleuro-peritoneal canal would lie close to the oesophagus and thus would not be visible through the usual thoracoscopic approach in the axilla. Its para-oesophageal position at the highest point of the cupola might explain why the small quantity of exudate and oil in the pleural cavity did not seep through into the abdomen, and possibly why the defect was not sealed by pleural reaction.

The diaphragm is derived from the septum transversum with additions from the body wall, meso-oesophagus, and pleuro-peritoneal membranes (Hamilton, Boyd, and Mossman, 1948). The membranes normally occlude the pleuro-peritoneal canals at the eighth week, but if closure is incomplete a defect persists, through which abdominal viscera may herniate if it is of sufficient size. On the right side such a fistula would lie dorsal and slightly posterior to the left triangular ligament of the liver, between the 
caudate lobe and the oesophagus. Diaphragmatic hernia is uncommon on the right side because of the presence of the liver, and thus a right-sided pleuro-peritoneal defect is usually symptomless. The preponderance in reported cases of spontaneous pneumothorax on the right complicating pneumoperitoneum treatment may be ascribed to the frequent presence of a hernia when the defect occurs on the left side, for in such cases pneumoperitoneum would not be considered. Boyd (1948) noted two cases, both right-sided, of persistent pleuro-peritoneal canal recorded in anatomical journals recently.

The fistula may be fully patent, in which case a spontaneous pneumothorax will immediately follow pneumoperitoneum induction, or it may be merely a potential communication, being normally occluded by loose adherence of its walls. It might then be forced open by excessive intraabdominal pressure, which would explain the sudden appearance of a pneumothorax some time after the pneumoperitoneum had been fully established. This hypothesis receives support by analogy with another complication of pneumoperitoneum, scrotal pneumatocele. Cases have been described by Besta (1934), Monto and Bradford (1943), Banyai (1946), Rogers and Garrett (1947), Nagley (1948), and Meltzer (1948). Air suddenly appears within the tunica vaginalis of the scrotum on one or both sides, either immediately or several weeks or months after induction. The pneumatocele results from abnormal persistence of a communication between the general peritoneal cavity and that part of it which accompanies the testis in its descent into the scrotum. Irritation by air of the serosal lining of the processus vaginalis, especially after application of pressure to the inguinal ring, may cause it to obliterate, for it frequently does not recur.

In the present case it is postulated that a slitlike pleuroperitoneal opening had persisted, lateral to the oesophagus and medial to the left triangular ligament of the liver. It was probably valvular and of considerable size.

\section{SUMMARY}

Cases described in the literature of spontaneous pneumothorax occurring during the course of artificial pneumoperitoneum treatment are reviewed, and a further example recorded.

It is suggested that most, if not all, of these cases are due to the abnormal persistence of a pleuroperitoneal canal. In the instance reported it was not possible to demonstrate directly the presence of such a congenital defect, but it could be inferred with considerable certainty.

I should like to thank Mr. Vernon Thompson for helpful suggestions, and Dr. Kenneth Perry for permission to publish the case history.

\section{REFERENCES}

Banyai, A. L. (1933). Amer. J. med. Sci., 186, 513. (1946). Pneumoperitoneum Treatment, London. and Jurgens, G. H. (1940). Amer. Rev. Tuberc., 42, 688.

Besta, B. (1934). Lotta controlla Tuberc., 5, 499.

Boyd, J. D. (1948). Personal communication.

Clifford-Jones, E., and MacDonald, N.(1943). Tubercle, $24,97$.

Edwards, P. W., and Logan, J. (1945). Tubercle, 26, 11. Hamilton, W. J., Boyd, J. D., and Mossman, H. W. (1948). Human Embryology. W. Heffer and Sons. Ltd., Cambridge.

Hamman, L. (1939). Ann. intern. Med., 13, 932.

Jones, R. (1945). Brit. med. J., 2, 530.

Keers, R. Y., Rigden, B. G., and Steen, P. (1947). Edinb. med. J., 54, 30. (1948). Brit.J. Tuberc., 42, 58.

Laird, R. (1945). Tubercle, 26, 149.

Macklin, C. C. (1937). Canad. med. Ass. J., 36, 414.

Mellies, C. J. (1939). J. Missouri med. Ass., 36, 430.

Meltzer, H. (1948). Brit. J. Tuberc., 42, 70.

Mitchell, R. S., et al. (1947). Amer. Rev. Tuberc., 55, 306.

Monto, R. W., and Bradford, H. A.(1943). Ibid., 47, 537.

Nagley, M. M. (1948). Brit. J. Tuberc., 42, 17.

Rilance, A. B., and Warring, F. C. (1944). Amer. Rev. Tuberc., 49, 353.

Rogers, W. N., and Garrett, J. V. (1947). Brit. J. Tuberc., 47, 537.

Simmonds, F. A. H. (1946). Lancet, 1, 530.

Smith, C. N. (1943). Brit. med. J., 2, 404.

Stokes, J. B. (1938). Illinois med. J., 73, 137.

Trimble, H. G., and others (1948). Amer. Rev. Tuberc., $57,433$.

Vajda, L. (1933). Z. Tuberk., 67, 371. 